\title{
Influência do teor de umidade na determinação do módulo de elasticidade de vigas de Pinus sp.
}

\author{
Influence of moisture content on the determination of \\ the modulus of elasticity of Pinus sp. beams
}
Pedro Gutemberg de Alcântara Segundinho Marcelo Rodrigo Carreira Adair José Regazzi Antonio Alves Dias

\section{Resumo}

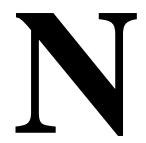

o processo de secagem da madeira o módulo de elasticidade estático não sofre alteração até o ponto de saturação das fibras (PSF); para teores de umidade abaixo do PSF, contudo, o módulo de elasticidade estático é afetado. O objetivo deste artigo foi investigar a influência do teor de umidade na obtenção do módulo de elasticidade de vigas de madeira serrada de Pinus sp. pelos ensaios de vibração transversal livre e flexão estática. Foram utilizadas 20 vigas com dimensões nominais de $5 \mathrm{~cm}$ x $10 \mathrm{~cm}$ x $200 \mathrm{~cm}$ originadas de plantios de reflorestamento do estado de São Paulo. Tais vigas foram extraídas de toras recém-abatidas e condicionadas em ambiente fechado para secagem com temperatura e umidade controladas. O módulo de elasticidade longitudinal aparente obtido pelo método de vibração transversal livre estabilizouse com umidade na faixa de variação do PSF, tal como ocorre no ensaio de flexão estática. Constatou-se que o ensaio de vibração transversal livre pode ser utilizado para obter o módulo de elasticidade longitudinal aparente em peças de madeira tanto com teor de umidade acima quanto abaixo do PSF.

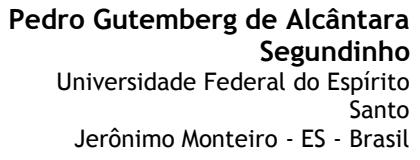

Marcelo Rodrigo Carreira
Universidade Tecnológica Federal do
Paraná
Campo Mourão - PR - Brasil

Adair José Regazzi Universidade Federal do Espírito
Santo Jerônimo Monteiro - ES - Brasil

Antonio Alves Dias Universidade de São Paulo São Carlos - SP - Brasil

Recebido em 10/10/15 Aceito em 26/11/16

Palavras-chave: Modelo de regressão segmentada. Madeira de Pinus sp. Ensaio não destrutivo. Ponto de saturação das fibras.

\section{Abstract}

In the wood drying process, the static modulus of elasticity does not change until the fibre saturation point (FSP) is reached; however, for lower than FSP moisture content, the static modulus of elasticity changes. The aim of this paper is to investigate the influence of moisture content on obtaining the modulus of elasticity of sawn wooden beams of Pinus sp. through free transverse vibration and static bending tests. We used 20 beams with nominal dimensions of $5 \mathrm{~cm} \times 10 \mathrm{~cm} \times 200$ cm from reforestation forests in the State of São Paulo. The beams were taken from newly harvested logs that were kept indoors for drying under controlled temperature and humidity. The apparent longitudinal modulus of elasticity, obtained through the free transverse vibration method, stabilized with humidity in the FSP variation range, as it occurs in the static bending test. The study showed that the free transverse vibration test can be used to obtain the apparent longitudinal modulus of elasticity from pieces of wood with a moisture content that is either above or below the FSP.

Keywords: Segmented regression model. Pinus sp. wood. Non-destructive testing. Fiber saturation point.
\end{abstract}




\section{Introdução}

No processo de secagem da madeira até o ponto de saturação das fibras (PSF) o módulo de elasticidade estático não sofre alteração e é admitido como constante. Para teores de umidade abaixo do PSF o módulo de elasticidade estático é afetado pelo teor de umidade (BODIG; JAYNE, 1982; LOGSDON, 1998).

O módulo de elasticidade na flexão pode ser avaliado por meio de ensaio estático ou ensaios dinâmicos, como ultrassom, ondas de tensão e vibração transversal livre. Entre esses métodos de avaliação não destrutiva, o ensaio de vibração transversal livre apresenta algumas vantagens em relação aos demais, como menor tempo para execução, repetitividade e possibilidade de obter o módulo de elasticidade levando-se em conta as deformações devidas ao cisalhamento. Deve-se destacar ainda que a técnica de vibração transversal livre tem demonstrado boa exatidão na estimativa da rigidez à flexão de peças estruturais de madeira serrada em razão da boa aderência entre o modelo matemático e o modelo físico do ensaio (BALLARIN; TARGA; PALMA, 2002). Tal aderência entre modelos também foi verificada por Carreira et al. (2003, 2012), Carreira, Segundinho e Dias (2012) e Candian e Sales (2009). Vale ressaltar que tais trabalhos realizados no Brasil por meio de vibração transversal foram em peças de madeira com teor de umidade abaixo do PSF.

A norma ASTM D 6874 (ASTM, 2003a) Standard Test Methods for Nondestructive Evaluation of Wood-Based Flexural Members Using Transverse Vibration - padroniza os procedimentos do ensaio de vibração transversal livre em vigas de madeira serrada. Segundo essa norma, o módulo de elasticidade dinâmico de vigas de madeira serrada com teor de umidade não superior a $22 \%$ pode ser corrigido empregando-se os procedimentos da norma ASTM D 2915 (ASTM, 2003b) - Practice for Evaluating Allowable Properties for Grades of Structural Lumber -, e para teores de umidade acima de $22 \%$ a norma ASTM D 6874 (ASTM, 2003a) não recomenda o emprego da norma ASTM D 2915 (ASTM, 2003b), deixando claro que qualquer ajuste do módulo de elasticidade acima desse teor de umidade deve ser documentado. A norma ASTM D 6874 (ASTM, 2003a) recomenda ainda que o emprego dos procedimentos da norma ASTM D 2915 (ASTM, 2003b) deve ser feito com certa cautela, uma vez que a aplicabilidade de tais procedimentos a resultados de ensaios dinâmicos não foi bem determinada.
Barret e Hong (2009) desenvolveram uma investigação experimental sobre o efeito do teor de umidade na medição do módulo de elasticidade dinâmico de vigas de madeira. Nesse estudo a água livre foi simulada com massas (moedas) distribuídas ao longo do comprimento. Os autores observaram que, adicionando-se massas para simular teores de umidade acima de $30 \%$, o módulo de elasticidade dinâmico permanece constante. Barret e Hong (2009) concluíram também que o procedimento da norma ASTM D 1990 (ASTM, 1997) - Standard Practice for Establishing Allowable Properties for VisuallyGraded Dimension Lumber from In-Grade Tests of Full-Size Specimens - para correção do módulo de elasticidade em função do teor de umidade pode ser usado para calcular o módulo de elasticidade dinâmico das vigas no teor de umidade de $15 \%$.

Unterwieser e Schickhofer (2010) estudaram a influência do teor de umidade no módulo de elasticidade dinâmico de vigas de madeira serrada e propuseram equações para correção do módulo de elasticidade da espécie Spruce para teores de umidade abaixo e acima do PSF.

A norma NBR 7190 (ABNT, 1997) recomenda a correção do módulo de elasticidade obtido por meio de ensaios estáticos para o teor de umidade de $12 \%$ e indica que não sejam utilizadas madeiras em aplicação estrutural com teores de umidade superiores a $20 \%$, pois a resistência e a rigidez da madeira sofrem variações durante o intervalo higroscópico até atingir o PSF, teor de umidade próximo de $30 \%$, a partir do qual se considera que o módulo de elasticidade permanece constante.

O objetivo do trabalho foi investigar a influência do teor de umidade na obtenção do módulo de elasticidade de vigas de madeira serrada de Pinus sp. pelos ensaios de vibração transversal livre e flexão estática simplesmente apoiada. Neste estudo ajustou-se um modelo de regressão segmentada para identificar o teor de umidade a partir do qual o módulo de elasticidade tende a ser constante.

\section{Material e métodos}

\section{Material}

Foram utilizadas 20 vigas de madeira serrada de Pinus sp. com dimensões nominais de $5 \mathrm{~cm} \times 10$ $\mathrm{cm}$ X $200 \mathrm{~cm}$ originadas de plantios de reflorestamento do estado de São Paulo. As vigas foram extraídas de toras recém-abatidas e foram condicionadas em uma sala fechada, cuja temperatura e umidade relativa do ar variaram de 21,4 a $24,6{ }^{\circ} \mathrm{C}$ e de $55 \%$ a $65 \%$ respectivamente. 
Acrescenta-se que questões como tipos de lenhos, juvenil e adulto, proporção de lenho inicial e tardio, número de anéis de crescimento, que interferem no módulo de elasticidade, foram desconsiderados neste estudo. Salienta-se que para trabalhos com madeira estrutural deve-se selecionar madeira de lenho adulto.

\section{Métodos}

Inicialmente foram estimados o teor de umidade e as densidades básica e aparente das vigas, a partir de corpos de prova extraídos delas, e medida a massa inicial de cada viga com o intuito de estimar o teor de umidade em cada observação.

A secagem das vigas foi feita de forma natural, e a cada variação de aproximadamente $5 \%$ no teor de umidade foram obtidas suas dimensões e massas e realizados os ensaios para a obtenção do módulo de elasticidade estático e das frequências naturais do primeiro modo de vibração por flexão das vigas. Tal procedimento foi realizado até as vigas atingirem a umidade de equilíbrio com o ambiente. Então foram extraídos 15 corpos de prova de cada viga para a determinação do teor de umidade médio de acordo com a norma NBR 7190 (ABNT, 1997) e obteve-se a massa seca das vigas a partir da determinação do teor de umidade final e correção das umidades dos ensaios, possibilitando calcular os teores de umidade efetivos em cada ensaio.

\section{Ensaio de flexão estática}

O ensaio de flexão estática foi realizado com o esquema de viga simplesmente apoiada com força concentrada na metade do vão da viga. Elas foram fletidas em relação ao eixo de menor inércia, com um vão de $185 \mathrm{~cm}$. A relação vão-altura foi igual a
37, e por essa razão o efeito do esforço cortante foi desprezado no cálculo dos módulos de elasticidade estático e dinâmico.

A força aplicada foi medida com um anel dinamométrico com capacidade de $4,7 \mathrm{kN}$, e os deslocamentos verticais na metade do comprimento das vigas foram medidos por meio de relógio comparador com resolução de $0,01 \mathrm{~mm}$.

Foi aplicada uma força crescente, tendo sido anotadas as forças necessárias para provocar deslocamentos verticais de $4 \mathrm{~mm}$ e $8 \mathrm{~mm}$. O módulo de elasticidade foi calculado a partir desses valores utilizando a Equação 1.

$$
\mathrm{MOE}_{s t}=\frac{\Delta F \cdot L^{3}}{48 \cdot \Delta v \cdot I}
$$

Em que:

$\mathrm{MOE}_{s t}$ é o módulo de elasticidade a flexão $(\mathrm{Pa})$

$\Delta F$ é o incremento de força $(\mathrm{N})$;

$L$ é o vão livre (m);

$\Delta v$ é o incremento no deslocamento vertical $\left(4 \cdot 10^{-3}\right.$ $\mathrm{m})$; e

I é o momento de inércia da seção transversal $\left(\mathrm{m}^{4}\right)$.

\section{Ensaio de vibração transversal livre}

O ensaio de vibração transversal livre foi realizado com excitação no plano vertical. As vigas foram suspensas por duas linhas de nylon, com diâmetro de $0,7 \mathrm{~mm}$, posicionadas nas coordenadas $0,224 l \mathrm{e}$ $0,776 l$, que correspondem aos pontos nodais do primeiro modo de flexão de uma viga em suspensão livre-livre, sendo $l$ o comprimento da viga. A Figura 1a ilustra uma visão geral do ensaio.

\section{Figura 1- Ensaio de vibração transversal livre nas vigas serradas de Pinus sp.}

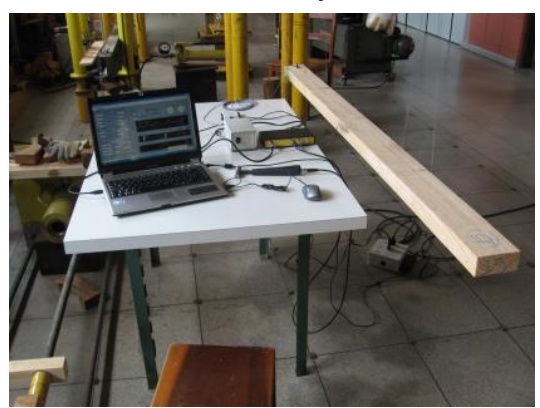

(a) Vista geral do ensaio

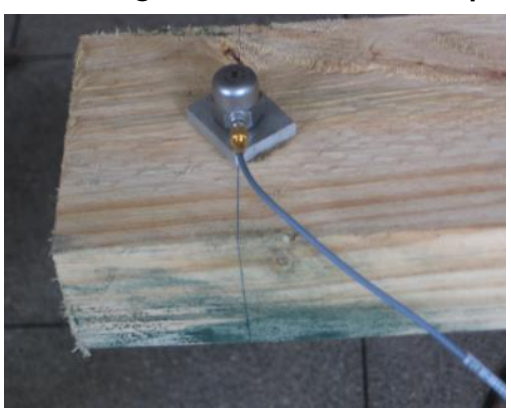

(b) Acelerômetro fixado na extremidade da viga

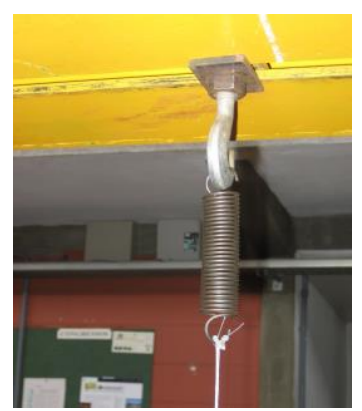

(c) Mola empregada para suspensão 
Os sinais do martelo e do acelerômetro foram enviados a um condicionador de sinais desenvolvido pelo primeiro autor e então foram convertidos em sinais digitais por uma placa USB 6009 da National Instruments Corporation. Os sinais foram adquiridos e analisados pelo programa Impact, também desenvolvido pelo primeiro autor, sendo a função de resposta em frequência (FRF) calculada com a média de 10 espectros. A identificação dos parâmetros modais foi feita com o programa Modal-Id, que faz a identificação multimodal pelo método da razão de polinômios.

O módulo de elasticidade dinâmico foi calculado pela Equação 2, obtida pela solução da equação de movimento de uma viga de Bernoulli para uma viga em suspensão livre-livre (CLOUGH; PENZIEN, 1995).

$\operatorname{MOE}_{v t}=\left(\frac{2 \cdot f_{1}}{\gamma_{1} \cdot \pi}\right)^{2} \cdot \frac{m \cdot l^{3}}{I}$

Em que:

$\mathrm{MOE}_{v t}$ é o módulo de elasticidade longitudinal obtido pelo método de vibração transversal livre (MPa);

$f_{1}$ é a primeira frequência de vibração transversal livre $(\mathrm{Hz}) ; \mathrm{e}$

$\gamma_{1}$ é obtido por meio da equação $(n+0,5)^{2}$, onde $n$ é igual a 1 (número do modo harmônico);

$m$ é a massa do corpo de prova $(\mathrm{kg})$;

$l$ é o comprimento da peça (m); e

I é o momento de inércia da seção transversal $\left(\mathrm{m}^{4}\right)$.

\section{Análise dos dados}

A análise dos dados foi realizada considerando-se o modelo estatístico polinomial quadrático com resposta constante (MPQC). É o mesmo modelo polinomial quadrático com resposta em platô, mas no estudo em questão a equação terá ponto de mínimo, não de máximo. $\mathrm{O}$ ajuste do modelo foi realizado a partir dos dados obtidos experimentalmente para as 20 vigas estudadas por meio do procedimento NLIN (PROC NLIN) do software estatístico SAS (Statistical Analysis Software) (STATISTICAL..., 2004).

\section{Modelo Polinomial Quadrático com Resposta Constante (MPQC)}

Consideraram-se os seguintes modelos (Equações 3 e 4):

$$
\begin{aligned}
& y=a+b x+c x^{2}+e \text { se } x<x_{0} \text { (quadrático) Eq. } 3 \\
& y=p+e \text { se } x \geq x_{0} \text { (constante) }
\end{aligned}
$$

Em que:

$y$ é o módulo de elasticidade longitudinal aparente $\left(E_{a}\right) ; \mathrm{e}$

$x$ é o teor de umidade.

A justaposição dos dois submodelos é concordante no ponto de interseção $x_{0}$. Dessa forma, para valores de $x$ menores que $x_{0}$, o modelo que descreve a resposta $y$ é uma função quadrática, e para valores de $x$ maiores ou iguais a $x_{0}$ o modelo é uma constante. Para estimação dos parâmetros, o modelo deve apresentar propriedades matemáticas adequadas, isto é, deve ser uma função contínua e diferençável em $x_{0}$. Essa condição implica que $x_{0}=-$ $b / 2 c$ e $p=a-\left(b^{2} / 4 c\right)$, em que $x_{0}$ é ponto de mínimo, o teor de umidade $x$ para um mínimo módulo de elasticidade longitudinal aparente e o ponto de interseção das duas linhas, e $p$ é a constante, sendo $a, b$ e $c$ os parâmetros do modelo a serem estimados. Assim, para valores de umidade maiores que $x_{0}$ o módulo de elasticidade estimado pelo modelo será a constante $p$.

\section{Avaliação do modelo}

Foi ajustado um modelo para cada uma das 20 vigas. A qualidade do ajustamento do modelo polinomial quadrático com resposta constante (MPQC) foi avaliada por meio dos seguintes critérios: (i) teste F para regressão; (ii) coeficiente de determinação $\left(R^{2}\right)$, obtido da seguinte forma (uma vez que o modelo inclui o intercepto) (Equação 5):

$R^{2}(\%)=\left(1-\frac{S Q R}{S Q T C}\right) \cdot 100$

Eq. 5

Em que $S Q R$ é a soma de quadrados do resíduo e $S Q T C$ é a soma de quadrados total corrigida pela média; e (iii) coeficiente de variação $(C V)$, definido por (Equação 6):

$C V(\%)=\frac{S_{y x}}{\bar{y}} \cdot 100$

Eq. 6

Em que $S_{y x}=\sqrt{\text { QMResíduo }}$ é o desvio padrão residual obtido da análise de variância da regressão (MPQC). O coeficiente de variação mede a dispersão relativa das observações, porque, por definição, é o cociente entre a medida da dispersão dos pontos em torno da curva ajustada $\left(S_{y x}\right)$ e o valor médio da variável dependente $(\bar{y})$. O resultado é tanto melhor quanto menor for o coeficiente de variação.

Para os testes estatísticos o nível de significância adotado foi de até $5 \%$ de probabilidade. Assim, para valor-P > 0,05, o resultado é declarado não significativo estatisticamente. 


\section{Resultados e discussão}

Os valores médios da densidade básica, densidade aparente e teor de umidade da madeira de Pinus sp. obtidos após a secagem natural são apresentados na Tabela 1.
Nas Figuras 2 e 3 são plotados os dados experimentais (módulo de elasticidade em função da umidade), valores estimados e pontos de interseção obtidos nos ensaios de flexão estática simplesmente apoiada e vibração transversal livre respectivamente.

Tabela 1 - Valores médios da densidade básica, densidade aparente e teor de umidade da madeira de Pinus sp. obtidos após a secagem natural

\begin{tabular}{l|c}
\hline Densidade básica $\left(\mathrm{g} / \mathrm{m}^{3}\right)$ & $0,49(0,06)^{*}$ \\
Densidade aparente $\left(\mathrm{g} / \mathrm{m}^{3}\right)$ & $0,56(0,07)^{*}$ \\
Teor de umidade $(\%)$ & $13,07(0,76)^{*}$ \\
\hline
\end{tabular}

Nota: *o valor entre parênteses é o desvio padrão.

Figura 2 - Dados experimentais (módulo de elasticidade em função da umidade), valores estimados e pontos de interseção obtidos no ensaio de flexão estática simplesmente apoiada

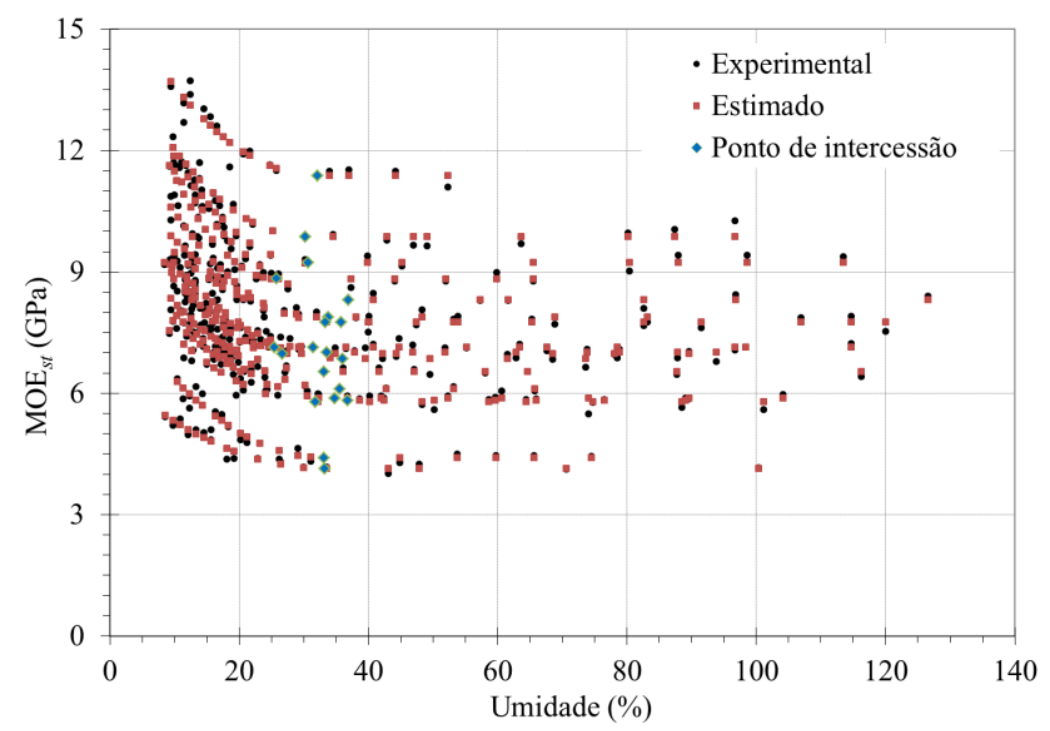

Figura 3 - Dados experimentais (módulo de elasticidade em função da umidade), valores estimados e pontos de interseção obtidos no ensaio de vibração transversal livre

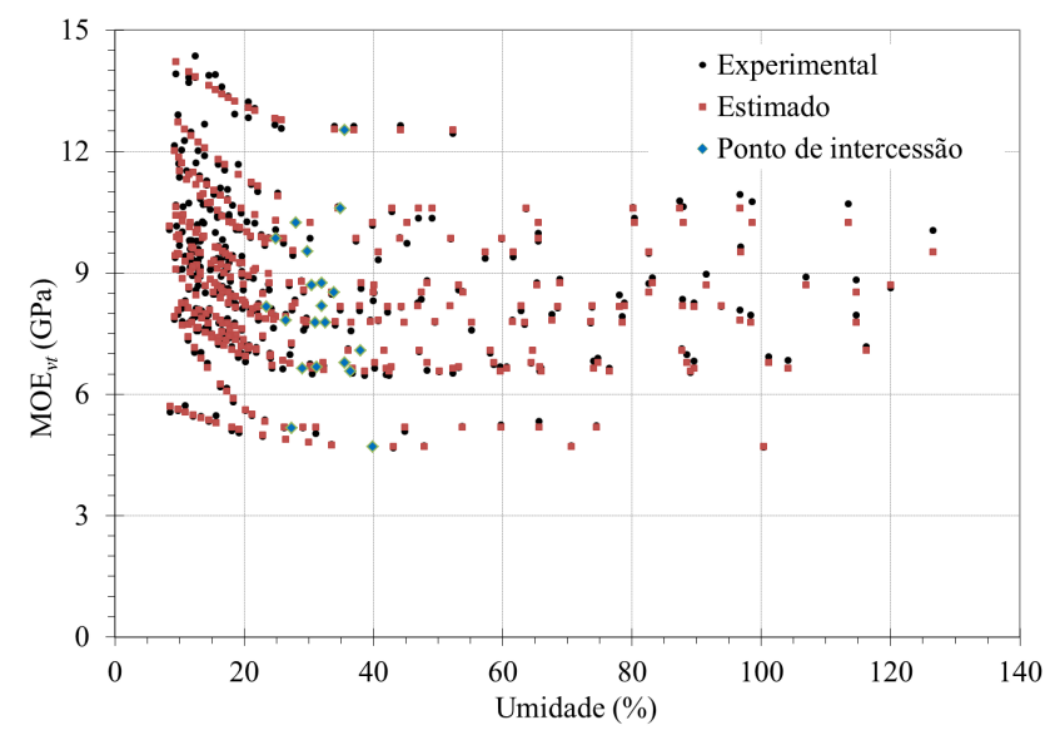


Na Tabela 2 está a análise de variância e teste F para regressão considerando o modelo polinomial quadrático com resposta constante para madeira de Pinus sp. com a carga aplicada formando ângulos quaisquer com as direções tangencial e radial no ensaio de flexão estática.

$\mathrm{Na}$ Tabela 3, por sua vez, estão as equações ajustadas para o modelo de regressão utilizado para madeira de Pinus sp. no ensaio de flexão estática, com os coeficientes de determinação $R^{2}$ (\%), coeficiente de variação $C V(\%)$, teor de umidade mínimo $x_{0}(\%)$ e a constante $p$. O teor de umidade a partir do qual não houve alteração significativa no módulo de elasticidade das vigas variou entre $25,42 \%$ e $36,95 \%$.

Tabela 2 - Análise de variância e teste F para regressão considerando o modelo polinomial quadrático com resposta constante para madeira de Pinus sp. com a carga aplicada formando ângulos quaisquer com as direções tangencial e radial no ensaio de flexão estática

\begin{tabular}{|c|c|c|c|c|c|}
\hline Vigas & $n$ & Fonte de variação & G. L. & Q. M. & $\mathbf{F}$ \\
\hline \multirow{2}{*}{1} & \multirow{2}{*}{18} & Regressão & 2 & 4,9035 & $125,09^{* *}$ \\
\hline & & Resíduo & 15 & 0,0392 & \\
\hline \multirow{2}{*}{2} & \multirow{2}{*}{18} & Regressão & 2 & 3,0196 & $46,31^{* *}$ \\
\hline & & Resíduo & 15 & 0,0652 & \\
\hline \multirow{2}{*}{3} & \multirow{2}{*}{19} & Regressão & 2 & 4,5203 & $162,60^{* * *}$ \\
\hline & & Resíduo & 16 & 0,0278 & \\
\hline \multirow{2}{*}{4} & \multirow{2}{*}{19} & Regressão & 2 & 5,2534 & $56,49^{* *}$ \\
\hline & & Resíduo & 16 & 0,0930 & \\
\hline \multirow{2}{*}{5} & \multirow{2}{*}{19} & Regressão & 2 & 4,2254 & $53,96^{* *}$ \\
\hline & & Resíduo & 16 & 0,0783 & \\
\hline \multirow{2}{*}{6} & \multirow{2}{*}{20} & Regressão & 2 & 6,0642 & $153,52^{\text {*** }}$ \\
\hline & & Resíduo & 17 & 0,0395 & \\
\hline \multirow{2}{*}{7} & \multirow{2}{*}{18} & Regressão & 2 & 8,9438 & $153,41^{* *}$ \\
\hline & & Resíduo & 15 & 0,0583 & \\
\hline \multirow{2}{*}{8} & \multirow{2}{*}{18} & Regressão & 2 & 10,5378 & $200,72^{\text {** }}$ \\
\hline & & Resíduo & 15 & 0,0525 & \\
\hline \multirow{2}{*}{9} & \multirow{2}{*}{19} & Regressão & 2 & 5,1881 & $220,77^{\text {*** }}$ \\
\hline & & Resíduo & 16 & 0,0235 & \\
\hline \multirow{2}{*}{10} & \multirow{2}{*}{18} & Regressão & 2 & 3,2821 & $119,78^{* *}$ \\
\hline & & Resíduo & 15 & 0,0274 & \\
\hline \multirow{2}{*}{11} & \multirow{2}{*}{20} & Regressão & 2 & 7,9446 & $99,43^{* *}$ \\
\hline & & Resíduo & 17 & 0,0799 & \\
\hline \multirow{2}{*}{12} & \multirow{2}{*}{18} & Regressão & 2 & 1,8129 & $88,43^{* *}$ \\
\hline & & Resíduo & 15 & 0,0205 & \\
\hline \multirow{2}{*}{13} & \multirow{2}{*}{18} & Regressão & 2 & 2,1358 & $133,49^{* *}$ \\
\hline & & Resíduo & 15 & 0,0160 & \\
\hline 14 & 18 & Regressão & 2 & 2,9753 & $89,08^{* *}$ \\
\hline 14 & 10 & Resíduo & 15 & 0,0334 & \\
\hline 15 & 10 & Regressão & 2 & 3,8809 & $102,13^{\text {** }}$ \\
\hline 15 & 19 & Resíduo & 16 & 0,0380 & \\
\hline 16 & 20 & Regressão & 2 & 7,0327 & $193,74^{* *}$ \\
\hline 10 & 20 & Resíduo & 17 & 0,0363 & \\
\hline & & Regressão & 2 & 4,4031 & $128,75^{* *}$ \\
\hline 17 & 21 & Resíduo & 18 & 0,0342 & \\
\hline 18 & 21 & Regressão & 2 & 5,5653 & $205,36^{* *}$ \\
\hline 10 & 21 & Resíduo & 18 & 0,0271 & \\
\hline 10 & & Regressão & 2 & 3,7467 & $37,69^{* *}$ \\
\hline 19 & 18 & Resíduo & 15 & 0,0994 & \\
\hline 20 & 20 & Regressão & 2 & 5,7293 & $116,45^{* *}$ \\
\hline 20 & 20 & Resíduo & 17 & 0,0492 & \\
\hline
\end{tabular}

Nota: **significativo em nível de $1 \%$ de probabilidade $(\mathrm{P}<0,01)$ e $n$ o número de observações em cada viga. 
Tabela 3 - Equações ajustadas para o modelo polinomial quadrático com resposta constante $(p)$, coeficiente de determinação $\left(R^{2}\right)$, coeficiente de variação $(C V)$, teor de umidade mínimo $\left(X_{0}\right)$ e a constante $(p)$ para madeira de Pinus sp. com a carga aplicada formando ângulos quaisquer com as direções tangencial e radial no ensaio de flexão estática

\begin{tabular}{|c|c|c|c|c|c|}
\hline Vigas & Equações ajustadas* & $R^{2}(\%)$ & $C V(\%)$ & $\mathrm{X}_{0}(\%)$ & $p$ (Gpa) \\
\hline 1 & $\hat{y}=10,2561-0,2335 x+0,00328 x^{2}$ & 94,34 & 2,79 & 35,58 & 6,10 \\
\hline 2 & $\hat{y}=9,2624-0,2189 x+0,00344 x^{2}$ & 86,06 & 4,05 & 31,80 & 5,78 \\
\hline 3 & $\hat{y}=11,3465-0,2679 x+0,00426 x^{2}$ & 95,32 & 2,15 & 31,43 & 7,14 \\
\hline 4 & $\hat{y}=16,0390-0,2905 x+0,00452 x^{2}$ & 87,59 & 2,48 & 32,12 & 11,37 \\
\hline 5 & $\hat{y}=11,7616-0,2300 x+0,00340 x^{2}$ & 87,08 & 3,24 & 33,81 & 7,87 \\
\hline 6 & $\hat{y}=11,4574-0,2643 x+0,00393 x^{2}$ & 94,75 & 2,55 & 33,62 & 7,01 \\
\hline 7 & $\hat{y}=13,2954-0,3318 x+0,00497 x^{2}$ & 95,34 & 2,73 & 33,37 & 7,76 \\
\hline 8 & $\hat{y}=14,2565-0,3219 x+0,00436 x^{2}$ & 96,39 & 2,40 & 36,95 & 8,31 \\
\hline 9 & $\hat{y}=11,8368-0,3641 x+0,00681 x^{2}$ & 96,50 & 2,00 & 26,73 & 6,97 \\
\hline 10 & $\hat{y}=12,2603-0,4029 x+0,00793 x^{2}$ & 94,11 & 2,14 & 25,42 & 7,14 \\
\hline 11 & $\hat{y}=15,5763-0,5230 x+0,01010 x^{2}$ & 92,12 & 2,95 & 25,82 & 8,83 \\
\hline 12 & $\hat{y}=6,5202-0,1432 x+0,00215 x^{2}$ & 92,19 & 3,10 & 33,25 & 4,14 \\
\hline 13 & $\hat{y}=9,7409-0,1938 x+0,00292 x^{2}$ & 94,68 & 1,80 & 33,14 & 6,53 \\
\hline 14 & $\hat{y}=11,0754-0,1860 x+0,00260 x^{2}$ & 92,23 & 2,18 & 35,78 & 7,75 \\
\hline 15 & $\hat{y}=9,6130-0,2148 x+0,00309 x^{2}$ & 92,74 & 2,99 & 34,79 & 5,88 \\
\hline 16 & $\hat{y}=14,9831-0,3752 x+0,00610 x^{2}$ & 95,80 & 1,90 & 30,73 & 9,22 \\
\hline 17 & $\hat{y}=8,4174-0,2425 x+0,00366 x^{2}$ & 93,46 & 3,62 & 33,11 & 4,40 \\
\hline 18 & $\hat{y}=9,8605-0,2196 x+0,00298 x^{2}$ & 95,81 & 2,49 & 36,80 & 5,82 \\
\hline 19 & $\hat{y}=10,6202-0,2098 x+0,00292 x^{2}$ & 83,41 & 4,17 & 35,99 & 6,84 \\
\hline 20 & $\hat{y}=14,6632-0,3166 x+0,00523 x^{2}$ & 93,20 & 2,10 & 30,28 & 9,87 \\
\hline
\end{tabular}

Nota: * $y$ é o módulo de elasticidade e $x$ é o teor de umidade (\%).

Analisando os resultados da Tabela 3, pode-se notar que os coeficientes de determinação para o modelo mostraram-se altos, com valores superiores a $83,41 \%$, além de coeficiente de variação com valores inferiores a $4,17 \%$, indicando que o modelo proporcionou bom ajuste dos dados.

$\mathrm{Na}$ Tabela 4 está a análise de variância e teste $\mathrm{F}$ para regressão considerando o modelo polinomial quadrático com resposta constante para madeira de Pinus sp. no ensaio de vibração transversal livre.

$\mathrm{Na}$ Tabela 5 estão as equações ajustadas para o modelo de regressão utilizado para madeira de Pinus sp. no ensaio de vibração transversal livre, com os coeficientes de determinação $\left(R^{2}\right)$, coeficiente de variação $(C V)$, teor de umidade mínimo $\left(x_{0}\right)$ e a constante $p$. O teor de umidade a partir do qual não houve alteração significativa no módulo de elasticidade das vigas variou entre $23,48 \%$ e $39,90 \%$.

Analisando os resultados da Tabela 5, pode-se notar que os coeficientes de determinação para o modelo mostraram-se altos, com valores superiores a $77,58 \%$, e coeficiente de variação com valores inferiores a $2,71 \%$. Logo, verifica-se que o modelo polinomial quadrático com resposta constante adotado no ajuste dos dados experimentais obtidos nos dois ensaios é adequado.

A partir dos dados das Tabelas 3 e 5, e considerando dados pareados, constatou-se que na comparação das médias de teor de umidade mínimo (média de $x_{0}$ no ensaio de flexão estática é igual a $32,5 \%$ e média de $x_{0}$ no ensaio de vibração transversal livre é igual a $31,6 \%$ ), pelo teste $t$ de Student, elas não diferiram estatisticamente (valor$\mathrm{P}=0,3109$ ). Nota-se que os valores apresentaram variação entre $23,48 \%$ e $39,90 \%$.

Tal como Oliveira e Sales (2005), utilizando aparelho de velocidade ultrassônica, observou-se uma inflexão em torno do PSF para várias espécies, entre essas pínus e eucaliptos, demonstrando que o efeito do teor de umidade abaixo do PSF é mais significativo do que acima desse ponto. Segundo Calegari et al. (2007), a grande influência do teor de umidade sobre o MOE, na faixa que corresponde à madeira completamente seca até o PSF, seria responsável pela variação da velocidade ultrassônica. 
Tabela 4 - Análise de variância e teste F para regressão considerando o modelo polinomial quadrático com resposta constante para madeira de Pinus sp. no ensaio de vibração transversal livre

\begin{tabular}{|c|c|c|c|c|c|}
\hline Vigas & $n$ & Fonte de variação & G. L. & Q. M. & $\mathbf{F}$ \\
\hline 1 & 18 & $\begin{array}{l}\text { Regressão } \\
\text { Resíduo }\end{array}$ & $\begin{array}{c}2 \\
15 \\
\end{array}$ & $\begin{array}{l}5,7578 \\
0,0175 \\
\end{array}$ & $329,02^{*}$ \\
\hline 2 & 18 & $\begin{array}{l}\text { Regressão } \\
\text { Resíduo } \\
\end{array}$ & $\begin{array}{c}2 \\
15 \\
\end{array}$ & $\begin{array}{l}1,3673 \\
0,0261 \\
\end{array}$ & $52,39^{*}$ \\
\hline 3 & 19 & $\begin{array}{l}\text { Regressão } \\
\text { Resíduo }\end{array}$ & $\begin{array}{c}2 \\
16\end{array}$ & $\begin{array}{l}3,2296 \\
0,0171\end{array}$ & $188,87^{*}$ \\
\hline 4 & 19 & $\begin{array}{l}\text { Regressão } \\
\text { Resíduo }\end{array}$ & $\begin{array}{c}2 \\
16 \\
\end{array}$ & $\begin{array}{l}2,7410 \\
0,0615\end{array}$ & $44,57^{*}$ \\
\hline 5 & 19 & $\begin{array}{l}\text { Regressão } \\
\text { Resíduo }\end{array}$ & $\begin{array}{c}2 \\
16\end{array}$ & $\begin{array}{l}2,8679 \\
0,0546\end{array}$ & $52,53^{*}$ \\
\hline 6 & 20 & $\begin{array}{l}\text { Regressão } \\
\text { Resíduo } \\
\end{array}$ & $\begin{array}{c}2 \\
17 \\
\end{array}$ & $\begin{array}{l}3,6625 \\
0,0154 \\
\end{array}$ & $237,82^{*}$ \\
\hline 7 & 18 & $\begin{array}{l}\text { Regressão } \\
\text { Resíduo }\end{array}$ & $\begin{array}{c}2 \\
15 \\
\end{array}$ & $\begin{array}{l}3,9739 \\
0,0482 \\
\end{array}$ & $82,45^{*}$ \\
\hline 8 & 18 & $\begin{array}{l}\text { Regressão } \\
\text { Resíduo }\end{array}$ & $\begin{array}{c}2 \\
15\end{array}$ & $\begin{array}{l}3,8576 \\
0,0364\end{array}$ & $105,98^{*}$ \\
\hline 9 & 19 & $\begin{array}{l}\text { Regressão } \\
\text { Resíduo }\end{array}$ & $\begin{array}{c}2 \\
16\end{array}$ & $\begin{array}{l}3,7240 \\
0,0274\end{array}$ & $135,91^{*}$ \\
\hline 10 & 18 & $\begin{array}{l}\text { Regressão } \\
\text { Resíduo }\end{array}$ & $\begin{array}{c}2 \\
15\end{array}$ & $\begin{array}{l}2,1029 \\
0,0251\end{array}$ & $83,78^{*}$ \\
\hline 11 & 20 & $\begin{array}{l}\text { Regressão } \\
\text { Resíduo }\end{array}$ & $\begin{array}{c}2 \\
17 \\
\end{array}$ & $\begin{array}{l}4,7126 \\
0,0796 \\
\end{array}$ & $59,20^{*}$ \\
\hline 12 & 18 & $\begin{array}{l}\text { Regressão } \\
\text { Resíduo }\end{array}$ & $\begin{array}{c}2 \\
15 \\
\end{array}$ & $\begin{array}{l}1,0528 \\
0,0069 \\
\end{array}$ & $152,58^{*}$ \\
\hline 13 & 18 & $\begin{array}{l}\text { Regressão } \\
\text { Resíduo }\end{array}$ & $\begin{array}{c}2 \\
15 \\
\end{array}$ & $\begin{array}{l}1,4955 \\
0,0055\end{array}$ & $271,91^{*}$ \\
\hline 14 & 18 & $\begin{array}{l}\text { Regressão } \\
\text { Resíduo }\end{array}$ & $\begin{array}{c}2 \\
15\end{array}$ & $\begin{array}{l}1,6693 \\
0,0294\end{array}$ & $56,78^{*}$ \\
\hline 15 & 19 & $\begin{array}{l}\text { Regressão } \\
\text { Resíduo } \\
\end{array}$ & $\begin{array}{c}2 \\
16 \\
\end{array}$ & $\begin{array}{l}1,9418 \\
0,0182 \\
\end{array}$ & $106,69^{*}$ \\
\hline 16 & 20 & $\begin{array}{l}\text { Regressão } \\
\text { Resíduo }\end{array}$ & $\begin{array}{c}2 \\
17 \\
\end{array}$ & $\begin{array}{l}2,4304 \\
0,0826 \\
\end{array}$ & $29,42^{*}$ \\
\hline 17 & 21 & $\begin{array}{l}\text { Regressão } \\
\text { Resíduo } \\
\end{array}$ & $\begin{array}{c}2 \\
18 \\
\end{array}$ & $\begin{array}{l}7,5247 \\
0,0082 \\
\end{array}$ & $917,65^{*}$ \\
\hline 18 & 21 & $\begin{array}{l}\text { Regressão } \\
\text { Resíduo }\end{array}$ & $\begin{array}{c}2 \\
18\end{array}$ & $\begin{array}{l}3,6588 \\
0,0125\end{array}$ & $292,70^{*}$ \\
\hline 19 & 18 & $\begin{array}{l}\text { Regressão } \\
\text { Resíduo }\end{array}$ & $\begin{array}{c}2 \\
15\end{array}$ & $\begin{array}{l}2,6342 \\
0,0510\end{array}$ & $51,65^{*}$ \\
\hline 20 & 20 & Regressão & $\frac{2}{17}$ & 5,7195 & $101,59^{*}$ \\
\hline
\end{tabular}

Nota: *significativo em nível de $1 \%$ de probabilidade $(\mathrm{P}<0,01)$ e $n$ o número de observações em cada viga. 
Tabela 5 - Equações ajustadas para o modelo polinomial quadrático com resposta constante, coeficiente de determinação $\left(R^{2}\right)$, coeficiente de variação $(C V)$, teor de umidade mínimo $\left(X_{0}\right)$ e a constante $(p)$ para madeira de Pinus sp. no ensaio de vibração transversal livre

\begin{tabular}{|c|c|c|c|c|c|}
\hline Vigas & Equações ajustadas* & $R^{2}(\%)$ & $C V(\%)$ & $x_{0}(\%)$ & $p(\mathbf{G P a})$ \\
\hline 1 & $\hat{y}=11,6187-0,3158 x+0,00505 x^{2}$ & 97,77 & 1,74 & 31,29 & 6,68 \\
\hline 2 & $\hat{y}=8,8567-0,1175 x+0,00165 x^{2}$ & 87,48 & 2,26 & 35,52 & 6,77 \\
\hline 3 & $\hat{y}=11,3837-0,2335 x+0,00377 x^{2}$ & 95,94 & 1,58 & 30,98 & 7,77 \\
\hline 4 & $\hat{y}=15,6518-0,1756 x+0,00247 x^{2}$ & 84,78 & 1,87 & 35,53 & 12,53 \\
\hline 5 & $\hat{y}=12,1194-0,2106 x+0,00329 x^{2}$ & 86,77 & 2,50 & 31,97 & 8,75 \\
\hline 6 & $\hat{y}=11,8371-0,2284 x+0,00357 x^{2}$ & 96,54 & 1,42 & 31,98 & 8,18 \\
\hline 7 & $\hat{y}=12,7506-0,2660 x+0,00437 x^{2}$ & 91,67 & 2,34 & 30,45 & 8,70 \\
\hline 8 & $\hat{y}=14,0312-0,3030 x+0,00508 x^{2}$ & 93,40 & 1,88 & 29,81 & 9,52 \\
\hline 9 & $\hat{y}=13,0362-0,4146 x+0,00883 x^{2}$ & 94,44 & 1,90 & 23,48 & 8,17 \\
\hline 10 & $\hat{y}=11,7025-0,2938 x+0,00555 x^{2}$ & 91,77 & 1,91 & 26,45 & 7,82 \\
\hline 11 & $\hat{y}=15,2873-0,4370 x+0,00876 x^{2}$ & 87,45 & 2,71 & 24,94 & 9,84 \\
\hline 12 & $\hat{y}=6,3069-0,0805 x+0,00101 x^{2}$ & 95,30 & 1,62 & 39,90 & 4,70 \\
\hline 13 & $\hat{y}=9,3756-0,1209 x+0,00159 x^{2}$ & 97,33 & 0,99 & 37,97 & 7,08 \\
\hline 14 & $\hat{y}=11,1439-0,1547 x+0,00228 x^{2}$ & 88,34 & 1,91 & 33,96 & 8,52 \\
\hline 15 & $\hat{y}=9,9393-0,2281 x+0,00393 x^{2}$ & 93,02 & 1,92 & 29,01 & 6,63 \\
\hline 16 & $\hat{y}=14,1099-0,2764 x+0,00494 x^{2}$ & 77,58 & 2,69 & 27,98 & 10,24 \\
\hline 17 & $\hat{y}=11,7578-0,4809 x+0,00878 x^{2}$ & 99,02 & 1,52 & 27,38 & 5,17 \\
\hline 18 & $\hat{y}=9,8687-0,1818 x+0,00250 x^{2}$ & 97,02 & 1,55 & 36,42 & 6,56 \\
\hline 19 & $\hat{y}=11,2564-0,2130 x+0,00326 x^{2}$ & 87,33 & 2,71 & 32,62 & 7,78 \\
\hline 20 & $\hat{y}=14,7031-0,2358 x+0,00338 x^{2}$ & 92,28 & 2,09 & 34,90 & 10,59 \\
\hline
\end{tabular}

Nota: *y é o módulo de elasticidade e $x$ é o teor de umidade (\%).

No estudo realizado por Gerhards (1982), a partir de diversos trabalhos, é confirmada a redução da resistência e do módulo de elasticidade da madeira com o acréscimo da umidade, até valores de teor de umidade próximos de $30 \%$, o que comprova a influência da umidade e da temperatura em várias propriedades mecânicas da madeira e, dessa forma, constata-se que os acréscimos de teores de umidade acima desse ponto não mais influenciam os parâmetros mecânicos.

Segundo Lima, Della Lucia e Vital (1986), o ponto de interseção encontrado, que relaciona as propriedades mecânicas da madeira, foi de $31,0 \%$ de umidade quando se estudou a influência da variação no teor de umidade da madeira de Eucalyptus saligna com 40 anos de idade.

\section{Conclusões}

O modelo polinomial quadrático com resposta constante mostrou-se adequado no ajuste dos dados. Logo, comprovou-se que esse modelo pode ser usado para descrever a variação do módulo de elasticidade aparente em função do teor de umidade tanto no ensaio de flexão estática simplesmente apoiada quanto no ensaio de vibração transversal livre.

O teor de umidade a partir do qual não há alteração significativa no módulo de elasticidade das vigas variou entre $25,42 \%$ e $36,95 \%$ no caso da flexão estática simplesmente apoiada e entre $23,48 \%$ e $39,90 \%$ no caso da vibração transversal livre. A análise estatística efetuada para o teor de umidade mínimo, considerando dados pareados, permite concluir pela equivalência entre as médias obtidas nos dois tipos de ensaio. O módulo de elasticidade longitudinal aparente obtido pelo método de vibração transversal livre estabilizou-se com umidade na faixa de variação do PSF. Para efeito de classificação estrutural comparativa entre peças de madeira, pode-se concluir que a obtenção do módulo de elasticidade longitudinal aparente com valores altos do teor de umidade, acima do PSF, não tem influência. 


\section{Referências}

AMERICAN SOCIETY OF TESTING AND MATERIAL. ASTM D 1990: standard practice for establishing allowable properties for visuallygraded dimension lumber from in-grade tests of full-size specimens. Philadelphia, 1997.

\section{AMERICAN SOCIETY OF TESTING AND} MATERIAL. ASTM D 6874: standard test method for nondestructive evaluation of woodbased flexural members using transverse vibration. Philadelphia, 2003a.

AMERICAN SOCIETY OF TESTING AND MATERIAL. ASTM D 2915: practice for evaluating allowable properties for grades of structural lumber. Philadelphia, $2003 \mathrm{~b}$.

ASSOCIAÇÃO BRASILEIRA DE NORMAS TÉCNICAS. NBR 7190: projeto de estruturas de madeira. Rio de Janeiro, 1997.

BALlARIN, A. W.; TARGA, L. A.; PALMA, H. A. L. Ensaios Não-Destrutivos de Vibração Transversal na Avaliação do Módulo de Elasticidade de Madeiras de Reflorestamento. In: ENCONTRO BRASILEIRO EM MADEIRAS E ESTRUTURAS DE MADEIRA, 8., Uberlândia, 2002. Anais... Uberlândia, 2002.

BARRET, J. D.; HONG, J. P. Moisture Content Adjustments for Dynamic Modulus of Elasticity of Wood Members. Wood Science and Technology, v. 44, n. 3, p. 485-495, 2009.

BODIG, J.; JAYNE, B. A. Mechanics of Wood and Wood Composites. New York: Van NostranReinhold, 1982.

CALEGARI, L. et al. Monitoramento do Teor de Umidade de Madeiras de Pinus elliottii Engelm e Eucalyptus grandis W. HILL ex Maiden, Sob Diferentes Temperaturas de Secagem, Através do Ultra-Som. Ciência Florestal, Santa Maria, v. 17, n. 4, p. 399-408, 2007.

CANDIAN, M.; SALES, A. Aplicação das Técnicas Não-Destrutivas de Ultra-Som, Vibração Transversal e Ondas de Tensão Para Avaliação de Madeira. Ambiente Construído, Porto Alegre, v. 9, n. 4, p. 83-98, out./dez. 2009.

CARREIRA, M. R. et al. Avaliação de Técnicas Não Destrutivas Aplicadas à Madeira de Pinus sp. In: CONFERÊNCIA PAN-AMERICANA DE ENSAIOS NÃO DESTRUTIVOS, 3., Rio de Janeiro, 2003. Anais... Rio de Janeiro, 2003.
CARREIRA, M. R. et al. Bending Stiffness Evaluation of Teca and Guajará Lumber Through Tests of Transverse and Longitudinal Vibration. Acta Scientiarum. Technology, v. 34, p. 27-32, 2012.

CARREIRA, M. R.; SEGUNDINHO, P. G. A.; DIAS, A. A. Determination of the E/G Ratio of Wood Logs Using Transverse Vibration. Wood Research, Bratislava, v. 57, p. 207-220, 2012.

CLOUGH, R. W.; PENZIEN, J. Dynamics of structures. 3. ed. Berkeley: Computers \& Structures, 1995.

GERHARDS, C. C. Effect of the Moisture Content and Temperature on the Mechanical Properties of Wood and Analysis of Immediate Effects. Wood and Fiber, v. 14, n. 1, p. 4-36, 1982.

LIMA, J. T.; DELLA LUCIA, R. M.; VITAL, B. R. Influência do teor de umidade nas propriedades mecânicas da madeira de Eucalyptus saligna Smith. Revista Árvore, v. 10, n. 1, p. 27-43, 1986.

LOGSDON, N. B. Influência da Umidade nas Propriedades de Resistência e Rigidez da Madeira. São Paulo, 1998. 174 f. Tese (Doutorado em Engenharia Civil) - Escola de Engenharia, Universidade de São Paulo, 1998.

OLIVEIRA, F. G. R.; SALES, A. Efeito da Densidade e do Teor de Umidade na Velocidade Ultra-Sônica da Madeira. Revista Minerva, v. 2, n. 1, p. 25-31, 2005.

STATISTICAL ANALYSIS SOFTWARE. SAS/STAT, Version 9.1, User's Guide, USA: Cary, North Carolina, 2004.

UNTERWIESER, H.; SCHICKHOFER, G. Influence of Moisture Content of Wood on Sound Velocity and Dynamic MOE of Natural Frequency- and Ultrasonic Runtime Measurement. European Journal of Wood and Wood Products, v. 69, n. 2, p. 171-181, 2010.

\section{Agradecimentos}

Os autores agradecem ao Conselho Nacional de Desenvolvimento Científico e Tecnológico (CNPq) e à Fundação de Amparo à Pesquisa do Espírito Santo (FAPES). 
Pedro Gutemberg de Alcântara Segundinho

Departamento de Ciências Florestais e da Madeira | Universidade Federal do Espírito Santo | Av. Gov. Lindemberg, 316, Centro | Jerônimo Monteiro - ES - Brasil | CEP 29550-000 | Tel.: (28) 3558-2515 | E-mail: p_gutemberg2001@yahoo.com.br

\section{Marcelo Rodrigo Carreira}

Departamento Acadêmico de Construção Civil | Universidade Tecnológica Federal do Paraná | Via Marginal Rosalina Maria dos Santos | Campus Campo Mourão, Área Urbanizada I | Campo Mourão - PR - Brasil | Caixa Postal 271 | CEP 87301-899 | Tel.: (44) 3518-1400 |

E-mail: carreira@utfpr.edu.br

Adair José Regazzi

Departamento de Ciências Florestais e da Madeira | Universidade Federal do Espírito Santo | Tel.: (31) 9617-1458 |

E-mail: adairreg@ufv.br

\section{Antonio Alves Dias}

Departamento de Engenharia de Estruturas, Escola de Engenharia | Universidade de São Paulo | Av. Trabalhador Sãocarlense, 400, Parque Arnold Schimidt | São Carlos - SP - Brasil | CEP 13566-590 | Tel.: (16) 3373-8207 | E-mail: dias@sc.usp.br

Revista Ambiente Construído

Associação Nacional de Tecnologia do Ambiente Construído

Av. Osvaldo Aranha, $99-3^{\circ}$ andar, Centro

Porto Alegre - RS - Brasil

CEP 90035-190

Telefone: +55 (51) 3308-4084

Fax: +55 (51) 3308-4054

www.seer.ufrgs.br/ambienteconstruido

E-mail: ambienteconstruido@ufrgs.br 